\title{
STRONG STATIONARY DUALITY AND ALGEBRAIC DUALITY FOR CONTINUOUS TIME MÖBIUS MONOTONE MARKOV CHAINS
}

\author{
Pan Zhao \\ Institute of Fundamental and Interdisciplinary Sciences, Beijing Union \\ University, Beijing 100101, P. R. China
}

\begin{abstract}
Under the assumption of Möbius monotonicity, we develop the theory of strong stationary duality for continuous time Markov chains on the finite partially ordered state space, we also construct a nonexplosive algebraic duality for continuous time Markov chains on $\mathbb{Z}_{+}^{d}$. Finally, we present an application to the two-dimensional birth and death chain.
\end{abstract}

Keywords: strong stationary times, strong stationary duality, algebraic duality, Möbius function, Möbius monotonicity.

${ }^{*}$ Corresponding author.

E-mail address: ldtzhaopan@buu.edu.cn (Pan Zhao).

Copyright @ 2021 Scientific Advances Publishers

2020 Mathematics Subject Classification: 06A06, 60J27, 60J28.

Submitted by Zeki Kasap.

Received October 27, 2021

This work is licensed under the Creative Commons Attribution International License (CC BY 3.0).

http://creativecommons.org/licenses/by/3.0/deed.en_US

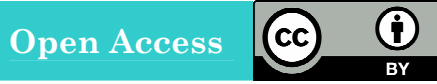




\section{Introduction}

A strong stationary time (SST) introduced by Aldous and Diaconis $[1,2]$ is a randomized stopping time $T$ for the discrete time chain $\mathbf{X}$ such that $X_{T}$ has the stationary distribution and is independent of $T$. The SST is a powerful tool to deal with the convergence to stationarity for a Markov chain. In order to get the SST, Diaconis and Fill [4] suggested a way to construct strong stationary duality (SSD), such that the absorption time of the SSD chain is equal in distribution to an SST for $\mathbf{X}$. Then one can bound the SST in the original chain through the absorption time. Especially, Diaconis and Fill showed a tractable case in [4] (Theorem 4.6) on the linearly ordered state space. In this case, they showed how to construct SSD, under the assumption of stochastic monotonicity. Diaconis and Fill [5] extended the theory of SSD for the discrete time chain on the countable state space. Fill [6] extended the theory of SSD for the continuous time chain.

Lorek and Szekli $[9,10]$ generalized the above tractable case for the discrete time chain, they considered the finite partially ordered state space instead of the linearly ordered state space. Since stochastic monotonicity is not sufficient in this case, they used Möbius monotone instead of stochastic monotone.

In this paper, we construct SSD for continuous time Möbius monotone Markov chains on the finite partially ordered state space. Also, we construct a nonexplosive algebraic duality for continuous time Markov

chains on $\mathbb{Z}_{+}^{d}$. In order to state conveniently, we introduce some symbols and definitions in [6].

Let $\mathbf{X}=\left(X_{t}\right)_{t \geq 0}$ be an ergodic continuous time Markov chain on the finite state space $E$, with initial distribution $\pi_{0}$, the conservative $Q$ matrix $Q$, the stationary distribution $\pi$, and the transition function $P(t)$. We write $\mathbf{X} \sim\left(\pi_{0}, Q, P(t)\right)$ for short below. Let $\mathbf{X}^{*} \sim\left(\pi_{0}^{*}, Q^{*}, P(t)\right)$ on 
the finite state space $E^{*}$. Assume $\mathbf{X}^{*}$ has an absorbing state $x_{a}$. Let $\Lambda(x, y), x \in E^{*}, y \in E$, be a link, or a transition kernel such that $\Lambda\left(x_{a}, \cdot\right)=\pi$. From [6], we know that $\mathbf{X}^{*}$ is an SSD for $\mathbf{X}$ with respect to $\Lambda$ if and only if the algebraic duality equations

$$
\pi_{0}=\pi_{0}^{*} \Lambda \text { and } \Lambda Q=Q^{*} \Lambda
$$

hold. Moreover, $\Lambda Q=Q^{*} \Lambda$ is equivalent to $\Lambda P(t)=P^{*}(t) \Lambda$ for all $t \geq 0$. In this case, the absorbing time $T_{x_{a}}^{*}$ that $\mathbf{X}^{*}$ hits $x_{a}$ is an SST for $\mathbf{X}$. For the countable state space $E$, Fill also gived SSD theory with some assumptions, which are called as "general setting" in [6]. However, if $E=\mathbb{Z}_{+}^{d}$, the assumptions are not easy to tractable. But we can construct a nonexplosive algebraic duality, which satisfies (1.1) and can also be used to study the convergence to stationarity for the Markov chain.

We will assume $E$ is a finite partially ordered state space or $\mathbb{Z}_{+}^{d}$ below. If $E$ is a finite partially ordered state space, define a partial order $\preceq$ on $E$. If $E=\mathbb{Z}_{+}^{d}$, define the natural partial order $\preceq$, i.e., for $x, y \in E$, $x \preceq y \Leftrightarrow x_{i} \leq y_{i}(\forall 1 \leq i \leq d)$.

From [8] and [9], we define $\downarrow$-Möbius monotonicity of a nonnegative function and a $Q$ matrix on $E$ as follows.

Definition 1.1. A nonnegative function $f$ on $E$ is $\downarrow$-Möbius monotone: if there exists a nonnegative function $m$ on $E$ such that $f(x)=\sum_{y \in E: y \succeq x} m(y)$ for any $x \in E$.

Define $\{x\}^{\downarrow}=\{y \in E: y \preceq x\}$ for any $x \in E$. Let $Q\left(x,\{y\}^{\downarrow}\right)=$ $\sum_{z \in E: z \preceq y} Q(x, z)$ for any $x, y \in E$. 
Definition 1.2. A $Q$ matrix $Q$ on $E \times E$ is $\downarrow$-Möbius monotone: if on $E \times E$, there exists a matrix $M$ which is nonnegative off the diagonal such that

$$
Q\left(x,\{y\}^{\downarrow}\right)=\sum_{z \in E: z \succeq x} M(z, y), \quad \forall x, y \in E .
$$

Definition 1.3. Let $\mathbf{X} \sim\left(\pi_{0}, Q, P(t)\right)$ on the countable state space. Suppose $P(t)$ is the minimal $Q$ process and let $\tau_{n}$ be the $n$-th jumping time of $\mathbf{X}$, then $Q$ is called nonexplosive if for any initial distribution, $\mathbb{P}\left(\lim _{n \rightarrow \infty} \tau_{n}=\infty\right)=1$.

Now we can state our main theorems.

Theorem 1.4. Let $\mathbf{X} \sim\left(\pi_{0}, Q, P(t)\right)$ be an ergodic continuous time Markov chain on a finite partially ordered state space $E=\left\{x^{1}, \ldots, x^{M}\right\}$, with a unique maximal state $x^{M}$ and the stationary distribution $\pi$. Suppose $Q$ is conservative, and define the time reversal of $Q$ as $\overleftarrow{Q}=\left(\overleftarrow{q}_{x, y}\right)$ with $\quad \overleftarrow{q}_{x, y}=\frac{\pi(y)}{\pi(x)} q, x$, let $H(x)=\sum_{y \in E: y \preceq x} \pi(y)$ and $g(x)=\frac{\pi_{0}(x)}{\pi(x)}$. Then there exists an SSD chain $\mathbf{X}^{*} \sim\left(\pi_{0}^{*}, Q^{*}, P^{*}(t)\right)$ on $E$ with the link kernel: $\Lambda(x, y)=I_{(y \preceq x)} \frac{\pi(y)}{H(x)}, x, y \in E$, if and only if the following two conditions hold:

(i) $g$ is $\downarrow$-Möbius monotone on $E$;

(ii) $\overleftarrow{Q}$ is $\downarrow$-Möbius monotone on $E$.

In this case, the SSD chain (for details, see Section 3) is uniquely determined by

$$
\frac{\pi_{0}(x)}{\pi(x)}=\sum_{y \in E: y \succeq x} \frac{\pi_{0}^{*}(y)}{H(y)} \text { and } \overleftarrow{Q}\left(y,\{x\}^{\downarrow}\right)=\sum_{z \in E: z \succeq y} \frac{H(x)}{H(z)} q_{x, z}^{*}
$$

for any $x, y \in E$. 
Theorem 1.5. Let $\mathbf{X} \sim\left(\pi_{0}, Q, P(t)\right)$ be an ergodic continuous time Markov chain on $E=\mathbb{Z}_{+}^{d}$, with the stationary distribution $\pi$. Suppose $Q$ is conservative and nonexplosive. Define the time reversal of $Q$ as $\overleftarrow{Q}=\left(\overleftarrow{q}_{x, y}\right)$ with $\overleftarrow{q}_{x, y}=\frac{\pi(y)}{\pi(x)} q_{y, x}$, let $H(x)=\sum_{y \in E: y \preceq x} \pi(y)$ and $g(x)=\frac{\pi_{0}(x)}{\pi(x)}$. Then there exists a nonexplosive algebraic duality chain $\mathbf{X}^{*} \sim\left(\pi_{0}^{*}, Q^{*}, P(t)\right)\left(P^{*}(t)\right.$ is the minimal $Q^{*}$ process $)$ on $E$ with the link kernel: $\Lambda(x, y)=I_{(y \preceq x)} \frac{\pi(y)}{H(x)}, x, y \in E$, if and only if the following three conditions hold:

(i) $g$ is $\downarrow$-Möbius monotone on $E$;

(ii) $\overleftarrow{Q}$ is $\downarrow$-Möbius monotone on $E$;

(iii) $Q^{*}$ is nonexplosive on $E$.

In this case, the nonexplosive algebraic duality chain (for details, see Section 3) is uniquely determined by

$$
\frac{\pi_{0}(x)}{\pi(x)}=\sum_{y \in E: y \succeq x} \frac{\pi_{0}^{*}(y)}{H(y)} \text { and } \overleftarrow{Q}\left(y,\{x\}^{\downarrow}\right)=\sum_{z \in E: z \succeq y} \frac{H(x)}{H(z)} q_{x, z}^{*}
$$

for any $x, y \in E$.

Let $\pi_{t}$ be the law of $X_{t}$. The variation distance is defined by $\left\|\pi_{t}-\pi\right\|$ $=\max _{A \subset E}\left|\pi_{t}(A)-\pi(A)\right|$. One advantage in establishing SSD is that useful bounds on variation distance can be obtained by stopping the process before absorption. Through this method, the early stopping theorem in [6] yielded useful bounds. Moreover, the early stopping theorem can apply to the algebraic duality. An argument similar to the early stopping theorem in [5] (Corollary 2.1) shows the following corollary, which transforms analysis of convergence rate for $\mathbf{X}$ into the first passage time for the dual chain. 
Corollary 1.6. For the chain $\left(\mathrm{X}^{*}, \mathrm{X}\right)$ of Theorem 1.4 or Theorem 1.5 , let $T$ be the first hitting time of $A \subset E$ for $\mathbf{X}^{*}$, where $A=\left\{x \in E: x \succeq x^{*}\right\}$, $x^{*} \in E$. Then

$$
\left\|\pi_{t}-\pi\right\| \leq\left(1-H\left(x^{*}\right)\right)+H\left(x^{*}\right) P\{T>t\}, \quad t \geq 0 .
$$

Here is an outline for the paper. Section 2 of this paper studies the Möbius function and Möbius monotonicity. Section 3 of this paper shows the proof of Theorem 1.4 and Theorem 1.5. Section 4 of this paper gives examples of the two-dimensional birth and death chain.

\section{Möbius Monotonicity}

Define $E$ as a finite partially ordered set or $\mathbb{Z}_{+}^{d}$. The zeta function $\zeta$ of $E$ is denoted by $\forall x, y \in E, \zeta(x, y)=1$ if $x \preceq y$ and $\zeta(x, y)=0$ otherwise. The inverse to $\zeta$ is the Möbius function $\mu$, which satisfies

$$
\sum_{z \in E: x \preceq z \preceq y} \mu(x, z) \zeta(z, y)=\sum_{z \in E: x \preceq z \preceq y} \zeta(x, z) \mu(z, y)=\delta(x, y),
$$

where $\delta(x, y)=1$ if $x=y$ and $\delta(x, y)=0$ otherwise. From Proposition 5 in [11], we obtain for any $x=\left(x_{1}, \ldots, x_{d}\right), y=\left(y_{1}, \ldots, y_{d}\right) \in \mathbb{Z}_{+}^{d}$,

$$
\begin{aligned}
\mu\left(\left(x_{1}, \ldots, x_{d}\right),\left(y_{1}, \ldots, y_{d}\right)\right) & \\
= & \left\{\begin{array}{cc}
(-1)^{\sum_{i=1}^{d}\left(y_{i}-x_{i}\right)}, & \text { if each } y_{i}-x_{i}=0 \text { or } 1, \\
0, & \text { otherwise }
\end{array}\right.
\end{aligned}
$$

With the help of Möbius function $\mu$, we will give criteria on the Möbius monotonicity of nonnegative functions and $Q$ matrices. From [9], we can obtain the following proposition: 
Proposition 2.1. If $E$ is a finite partially ordered set, then a nonnegative function $f$ on $E$ is $\downarrow$-Möbius monotone if and only if $\sum_{z \in E: z \succeq y}$ $\mu(y, z) f(z) \geq 0$ for any $y \in E$.

Proposition 2.2. If $E$ is a finite partially ordered set, then a $Q$ matrix $Q \quad$ on $E \times E$ is $\downarrow$-Möbius monotone if and only if $\sum_{z \in E: z \succeq y}$ $\mu(y, z) Q\left(z,\{x\}^{\downarrow}\right) \geq 0$ for any $x, y \in E$ and $x \neq y$.

Proof. Suppose $Q$ is $\downarrow$-Möbius monotone, then from Definition 1.2, there exists a matrix $M$ which is nonnegative off the diagonal such that

$$
Q\left(z,\{x\}^{\downarrow}\right)=\sum_{z^{\prime} \in E: z^{\prime} \succeq z} M\left(z^{\prime}, x\right), \quad \forall x, z \in E .
$$

Since $E$ is finite and (2.1), we can get

$$
\begin{aligned}
\sum_{z \in E: z \succeq y} \mu(y, z) Q\left(z,\{x\}^{\downarrow}\right) & =\sum_{z \in E: z \succeq y} \mu(y, z) \sum_{z^{\prime} \in E: z^{\prime} \succeq z} M\left(z^{\prime}, x\right) \\
& =\sum_{z^{\prime} \in E: z^{\prime} \succeq y} M\left(z^{\prime}, x\right) \sum_{z \in E: y \preceq z \preceq z^{\prime}} \mu(y, z) \\
& =\sum_{z^{\prime} \in E: z^{\prime} \succeq y} M\left(z^{\prime}, x\right) \delta\left(y, z^{\prime}\right)=M(y, x) \geq 0, \quad x \neq y .
\end{aligned}
$$

Conversely, let $M(z, y)=\sum_{z^{\prime} \in E: z^{\prime} \succeq z} \mu\left(z, z^{\prime}\right) Q\left(z^{\prime},\{y\}^{\downarrow}\right)$ for any $z, y \in E$, then $M$ is nonnegative off the diagonal. Since $E$ is finite and (2.1), we can obtain

$$
\begin{aligned}
\sum_{z \in E: z \succeq x} M(z, y) & =\sum_{z \in E: z \succeq x} \sum_{z^{\prime} \in E: z^{\prime} \succeq z} \mu\left(z, z^{\prime}\right) Q\left(z^{\prime},\{y\}^{\downarrow}\right) \\
& =\sum_{z^{\prime} \in E: z^{\prime} \succeq x} Q\left(z^{\prime},\{y\}^{\downarrow}\right) \sum_{z \in E: x \preceq z \preceq z^{\prime}} \mu\left(z, z^{\prime}\right) \\
& =\sum_{z^{\prime} \in E: z^{\prime} \succeq x} Q\left(z^{\prime},\{y\}^{\downarrow}\right) \delta\left(x, z^{\prime}\right)=Q\left(x,\{y\}^{\downarrow}\right) .
\end{aligned}
$$


Thus $Q$ is $\downarrow$-Möbius monotone.

Note that, if $E=\mathbb{Z}_{+}^{d}$, then $\lim _{x \rightarrow \infty} f(x)=0$ is to say for every $\epsilon>0$, there is a finite set $A \subset E$ such that $|f(x)| \leq \epsilon$ for any $x \in E \backslash A$. Then from [7] (Proposition 2.4 and Remark 2.5), we can get the following proposition.

Proposition 2.3. If $E=\mathbb{Z}_{+}^{d}$, then a nonnegative function $f$ on $E$ is $\downarrow$-Möbius monotone if and only if $\lim _{x \rightarrow \infty} f(x)=0$ and $\sum_{z \in E: z \succeq y}$ $\mu(y, z) f(z) \geq 0$ for any $y \in E$.

Proposition 2.4. If $E=\mathbb{Z}_{+}^{d}$, then a $Q$ matrix $Q$ on $E \times E$ is $\downarrow$-Möbius monotone if and only if $\lim _{x \rightarrow \infty} q_{x, y}=0$ for any $y \in E$, and $\sum_{z \in E: z \succeq y} \mu(y, z) Q\left(z,\{x\}^{\downarrow}\right) \geq 0$ for any $x, y \in E$ and $x \neq y$.

Proof. Suppose $Q$ is $\downarrow$-Möbius monotone, then from Definition 1.2, there exists a matrix $M$ which is nonnegative off the diagonal such that

$$
Q\left(x,\{z\}^{\downarrow}\right)=\sum_{y \in E: y \succeq x} M(y, z), \quad \forall x, z \in E .
$$

We will prove $\lim _{x \rightarrow \infty} Q\left(x,\{z\}^{\downarrow}\right)=\lim _{x \rightarrow \infty} \sum_{y \in E: y \succeq x} M(y, z)=0$. Since for fixed $z \in E$,

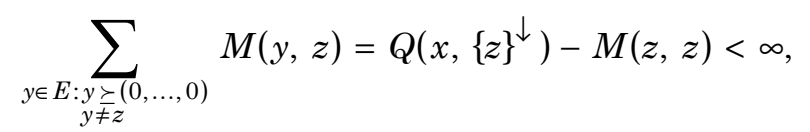

we have $\lim _{n \rightarrow \infty} \sum_{y \neq z, y \in E \backslash A_{n}} M(y, z)=0$, where $A_{n}=\{(n, \ldots, n)\}^{\downarrow}$, that is to say for any $\varepsilon>0$, there exists $N \geq 0$, such that when $n \geq N$, we have $\sum_{y \neq z, y \in E \backslash A_{n}} M(y, z) \leq \varepsilon$. Then, for any $\varepsilon>0$, for enough large $M \geq 0$, there exists $A_{M}$ such that when $x \in E \backslash A_{M}$, we have $z \nsucceq x$ and

$$
\sum_{y \in E: y \succeq x} M(y, z) \leq \sum_{y \neq z, y \in E \backslash A_{M}} M(y, z) \leq \varepsilon .
$$


Therefore, $\lim _{x \rightarrow \infty} \sum_{y \in E: y \succeq x} M(y, z)=\lim _{x \rightarrow \infty} Q\left(x,\{z\}^{\downarrow}\right)=0$. Also, since

$$
\begin{aligned}
\sum_{z \in E: z \preceq y} Q\left(x,\{z\}^{\downarrow}\right) \mu(z, y) & =\sum_{z \in E: z \preceq y} \sum_{z^{\prime} \in E: z^{\prime} \preceq z} q_{x, z^{\prime}} \mu(z, y) \\
& =\sum_{z^{\prime} \in E: z^{\prime} \preceq y} q_{x, z^{\prime}} \sum_{z \in E: z^{\prime} \preceq z \preceq y} \mu(z, y) \\
& =\sum_{z^{\prime} \in E: z^{\prime} \preceq y} q_{x, z^{\prime}} \delta_{z^{\prime}, y}=q_{x, y} .
\end{aligned}
$$

We get $\lim _{x \rightarrow \infty} q_{x, y}=0$. By using (2.1), we obtain

$$
\begin{aligned}
\sum_{z \in E: z \succeq y} \mu(y, z) Q\left(z,\{x\}^{\downarrow}\right) & =\sum_{z \in E: z \succeq y} \mu(y, z) \sum_{z^{\prime} \in E: z^{\prime} \succeq z} M\left(z^{\prime}, x\right) \\
& =\sum_{z^{\prime} \in E^{\prime}: z^{\prime} \succeq y} M\left(z^{\prime}, x\right) \sum_{z \in E: y \preceq z \preceq z^{\prime}} \mu(y, z) \\
& =\sum_{z^{\prime} \in E: z^{\prime} \succeq y} M\left(z^{\prime}, x\right) \delta\left(y, z^{\prime}\right)=M(y, x) \geq 0, \quad x \neq y .
\end{aligned}
$$

The second equality above is followed by the dominated convergence theorem. Therefore, $\sum_{z \in E: z \succeq y} \mu(y, z) Q\left(z,\{x\}^{\downarrow}\right) \geq 0$ for any $x \neq y$.

Conversely, let $M(z, y)=\sum_{z^{\prime} \in E: z^{\prime} \succeq z} \mu\left(z, z^{\prime}\right) Q\left(z^{\prime},\{y\}^{\downarrow}\right)$, for any $z, y \in E$. We need to prove $Q\left(x,\{y\}^{\downarrow}\right)=\sum_{z \in E: z \succeq x} M(z, y)$ for any $x, y \in E$. For convenience, we take $x=(0, \ldots, 0)$, and the proof is similar for any other $x \in E$. Define $e_{i}=(0, \ldots, 0,1,0, \ldots, 0)$ with 1 at the $i$-th coordinate, for convenience, we define $\sum_{y=x}^{z}=\sum_{y: x \preceq y \preceq z}$. Then by using (2.2), we get 


$$
\begin{aligned}
\sum_{z \in E: z \succeq(0, \ldots, 0)} M(z, y)= & \lim _{n \rightarrow \infty} \sum_{z=(0, \ldots, 0)}^{(n, \ldots, n)} M(z, y) \\
= & \lim _{n \rightarrow \infty} \sum_{z=(0, \ldots, 0)}^{(n, \ldots, n), z+(1, \ldots, 1)} \sum_{z^{\prime}=z} \mu\left(z, z^{\prime}\right) Q\left(z^{\prime},\{y\}^{\downarrow}\right) \\
= & \lim _{n \rightarrow \infty}\left(Q\left((0, \ldots, 0),\{y\}^{\downarrow}\right)+\sum_{i=1}^{d}(-1) Q\left((n+1) e_{i},\{y\}^{\downarrow}\right)\right. \\
& +\sum_{\substack{i \neq j \\
1 \leq i, j \leq d}}(-1)^{2} \cdot Q\left((n+1) e_{i}+(n+1) e_{j},\{y\}^{\downarrow}\right) \\
& \left.+\ldots+(-1)^{d} Q\left((n+1, \ldots, n+1),\{y\}^{\downarrow}\right)\right) \\
= & Q\left((0, \ldots, 0),\{y\}^{\downarrow}\right) .
\end{aligned}
$$

A similar proof of Lemma 2.4 in [12] shows that, for any $y \in E, \lim _{x \rightarrow \infty}$ $q_{x, y}=0$ implies $\lim _{x \rightarrow x^{*}} q_{x, y}=0$ for any $x^{*} \in \overline{\mathbb{Z}}_{+}^{d} \backslash E$ with $\overline{\mathbb{Z}_{+}}=\mathbb{Z}_{+}$ $\bigcup\{\infty\}$. Thus, the last equality above is followed by $\lim _{x \rightarrow \infty} q_{x, y}=0$ for any $y \in E$.

\section{Proof of Theorem 1.4 and Theorem 1.5}

With the help of Proposition 2.1 and Proposition 2.2, the proof of Theorem 1.4 is similar to that of Theorem 1.5 . Thus, we now only prove Theorem 1.5, using the Propositions 2.3 and 2.4.

Proof of Theorem 1.5. Let $E=\mathbb{Z}_{+}^{d}$, from [6], to prove the existence of nonexplosive algebraic duality, we need to check the algebraic duality equations

$$
\pi_{0}=\pi_{0}^{*} \Lambda, \quad \Lambda Q=Q_{0}^{*} \Lambda
$$


Also, we need to check that $Q^{*}$ is nonexplosive on $E$. As for $\pi_{0}=\pi_{0}^{*} \Lambda$, we have

$$
g(x)=\frac{\pi_{0}(x)}{\pi(x)}=\sum_{y \in E: y \succeq x} \frac{\pi_{0}^{*}(y)}{H(y)}, \quad \forall x \in E .
$$

Then we get $\pi_{0}=\pi_{0}^{*} \Lambda$ has a nonnegative solution if and only if $g$ is $\downarrow$-Möbius monotone, which from Proposition 2.3 is equivalent to $\lim _{x \rightarrow \infty} g(x)=0$ and $\sum_{z \in E: z \succeq y} \mu(y, z) g(z) \geq 0$, for any $y \in E$. Also, in this case, we get

$$
\pi_{0}^{*}(y)=H(y) \sum_{z \in E: z \succeq y} \mu(y, z) g(z) \geq 0, \quad \forall y \in E .
$$

From $\pi_{0}=\pi_{0}^{*} \Lambda$, it is clear that the solution sums to unity. Therefore, $\pi_{0}^{*}$ is a probability vector.

As for $\Lambda Q=Q^{*} \Lambda$, we have

$$
\sum_{z \in E} \Lambda(x, z) q_{z, y}=\sum_{z \in E} q_{x, z}^{*} \Lambda(z, y), \quad \forall x, y \in E,
$$

that is,

$$
\frac{1}{H(x)} \sum_{z \in E: z \preceq x} \frac{\pi(z)}{\pi(y)} q_{z, y}=\sum_{z \in E: z \succeq y} \frac{q_{x, z}^{*}}{H(z)} .
$$

Using $\frac{\pi(z)}{\pi(y)} q_{z, y}=\overleftarrow{q}_{y, z}$, we have

$$
\overleftarrow{Q}\left(y,\{x\}^{\downarrow}\right)=\sum_{z \in E: z \succeq y} \frac{H(x)}{H(z)} q_{x, z}^{*}, \quad \forall x, y \in E .
$$

Then we get $\Lambda Q=Q^{*} \Lambda$ has a solution which is nonnegative off the diagonal, if and only if $Q$ is $\downarrow$-Möbius monotone, which from Proposition 
2.4 is equivalent to $\lim _{x \rightarrow \infty} \overleftarrow{q}_{x, y}=0$ for any $y \in E$, and $\sum_{z \in E: z \succeq y}$ $\mu(y, z) \overleftarrow{Q}\left(z,\{x\}^{\downarrow}\right) \geq 0$ for any $x \neq y$. Also, in this case, we get

$$
q_{x, y}^{*}=\frac{H(y)}{H(x)} \sum_{z \in E: z \succeq y} \mu(y, z) \overleftarrow{Q}\left(z,\{x\}^{\downarrow}\right), \quad \forall x, y \in E .
$$

We now prove $Q^{*}$ is a conservative $Q$ matrix. From $\Lambda Q=Q^{*} \Lambda$, we have

$$
\sum_{z \in E} \Lambda(x, z) q_{z, y}=\sum_{z \in E} q_{x, z}^{*} \Lambda(z, y), \quad \forall x, y \in E .
$$

If $x \in E$, since $\{y \in E: y \preceq x\}$ is finite and $Q$ is conservative, we get

$$
\sum_{y \in E} \sum_{z \in E} \Lambda(x, z) q_{z, y}=\sum_{z \in E} \Lambda(x, z) \sum_{y \in E} q_{z, y}=0
$$

and

$$
\sum_{y \in E} \sum_{z \in E} q_{x, z}^{*} \Lambda(z, y)=\sum_{z \in E} q_{x, z}^{*} \sum_{y \in E} \Lambda(z, y)=\sum_{z \in E} q_{x, z}^{*}
$$

So $\sum_{z \in E} q_{x, z}^{*}=0$ for any $x \in E$. Since for any $x \neq y$,

$$
q_{x, y}^{*}=\frac{H(y)}{H(x)} \sum_{z \in E: z \succeq y} \mu(y, z) \overleftarrow{Q}\left(z,\{x\}^{\downarrow}\right) \geq 0
$$

we get $Q^{*}$ is a conservative $Q$ matrix.

\section{A Two-Dimensional Birth and Death Chain}

Assume $E=\{0,1, \ldots, N\}^{2}$ or $\mathbb{Z}_{+}^{2}, e_{1}=(1,0), e_{2}=(0,1)$. The $Q$ matrix of the two-dimensional birth and death chain $\mathbf{X}=\left(X_{t}\right)_{t \geq 0}$ is given as follows; for each $x=\left(x_{1}, x_{2}\right), y=\left(y_{1}, y_{2}\right) \in E$, let 


$$
q_{x, y}= \begin{cases}b_{i}, & \text { if } y=x+e_{i}, \\ a_{i}, & \text { if } y=x-e_{i}, \\ -\sum_{z \neq x} q_{x, z}, & \text { if } y=x \\ 0 & \text { else, }\end{cases}
$$

where $a_{i}>0, b_{i}>0$, and $a_{i} \neq b_{i}, i=1,2$. As is well known, $\mathbf{X}$ is time reversible, that is $\overleftarrow{Q}=Q$, and it has the unique stationary distribution: for any $x=\left(x_{1}, x_{2}\right) \in E$,

$$
\pi\left(x_{1}, x_{2}\right)=c\left(\frac{b_{1}}{a_{1}}\right)^{x_{1}}\left(\frac{b_{2}}{a_{2}}\right)^{x_{2}}
$$

with $c$ a normalizing constant.

Theorem 4.1. Assume $E=\{0,1, \ldots, N\}^{2}$, let $\mathbf{X} \sim\left(\pi_{0}, Q, P(t)\right)$ be a twodimensional birth and death chain with $Q$ given in (4.1), $a_{i}>0, b_{i}>0$, and $a_{i} \neq b_{i}, i=1,2$. Assume $\mathbf{X}$ starts at $(0,0)$, then there exists an SSD chain $\mathbf{X}^{*} \sim\left(\pi_{0}^{*}, Q^{*}, P^{*}(t)\right)\left(P^{*}(t)\right.$ is the $Q^{*}$ process $)$ starting at $(0,0)$ with the link kernel: $\Lambda(x, y)=I_{(y \preceq x)} \frac{\pi(y)}{H(x)}$, which is an absorbing Markov chain (with $(N, N)$ being the absorbing state) on $E$, with the following $Q$ matrix (for $\left.x=\left(x_{1}, x_{2}\right), y=\left(y_{1}, y_{2}\right) \in E\right)$, 


$$
q_{x, y}^{*}= \begin{cases}\frac{1-\left(b_{i} / a_{i}\right)^{x_{i}+2}}{1-\left(b_{i} / a_{i}\right)^{x_{i}+1}} a_{i}, & \text { if } y=x+e_{i}, \\ \frac{1-\left(b_{i} / a_{i}\right)^{x_{i}}}{1-\left(b_{i} / a_{i}\right)^{x_{i}+1}} b_{i}, & \text { if } y=x-e_{i}, x_{i} \neq N, \\ -\sum_{i=1}^{2}\left(a_{i}+b_{i}\right), & \text { if } y=x, x_{1} \neq N, x_{2} \neq N, \\ -a_{2}-b_{2}, & \text { if } y=x, x_{1}=N, x_{2} \neq N, \\ -a_{1}-b_{1}, & \text { if } y=x, x_{1} \neq N, x_{2}=N, \\ 0 & \text { else. }\end{cases}
$$

Theorem 4.2. Assume $E=\mathbb{Z}_{+}^{2}$, let $\mathbf{X} \sim\left(\pi_{0}, Q, P(t)\right)$ be a twodimensional birth and death chain with $Q$ given in (4.1), $a_{i}>0, b_{i}>0$, and $a_{i} \neq b_{i}, i=1,2$. Suppose $Q$ is nonexplosive. Assume $\mathbf{X}$ starts at $(0,0)$, then there exists a nonexplosive algebraic duality chain $\mathbf{X}^{*} \sim\left(\pi_{0}^{*}, Q^{*}, P^{*}(t)\right)\left(P^{*}(t)\right.$ is the minimal $Q^{*}$ process $)$ on $E$ with the link kernel: $\Lambda(x, y)=I_{(y \preceq x)} \frac{\pi(y)}{H(x)}$, which starts at $(0,0)$, with the following $Q$ matrix (for $\left.x=\left(x_{1}, x_{2}\right), y=\left(y_{1}, y_{2}\right) \in E\right)$,

$$
q_{x, y}^{*}= \begin{cases}\frac{1-\left(b_{i} / a_{i}\right)^{x_{i}+2}}{1-\left(b_{i} / a_{i}\right)^{x_{i}+1}} a_{i}, & \text { if } y=x+e_{i}, \\ \frac{1-\left(b_{i} / a_{i}\right)^{x_{i}}}{1-\left(b_{i} / a_{i}\right)^{x_{i}+1}} b_{i}, & \text { if } y=x-e_{i}, \\ -\sum_{i=1}^{2}\left(a_{i}+b_{i}\right), & \text { if } y=x, \\ 0 & \text { else. }\end{cases}
$$

We will only prove Theorem 4.2. With the help of Theorem 1.4, Theorem 4.1 can be proved similarly. 
Proof of Theorem 4.2. Since $H(x)=\sum_{y \in E: y \preceq x} \pi(y)$, we have $H(x)=$ $c\left(1-b_{1} / a_{1}\right)^{-1}\left(1-b_{2} / a_{2}\right)^{-1}$. Define $g(x)=\pi_{0}(x) / \pi(x)$. Assume the chain starts at $(0,0)$, then $g(x)=\pi_{0}(x) / \pi(x)$ is $\downarrow$-Möbius monotone. To prove the existence of the nonexplosive algebraic duality chain, from the proof of Theorem 1.5, we need to show that

$$
\left\{\begin{array}{l}
\text { (i) } \lim _{x \rightarrow \infty} g(x)=0 \text {, and } \sum_{z \in E: z \succeq y} \mu(y, z) g(z) \geq 0, \quad y \in E, \\
\text { (ii) } \lim _{x \rightarrow \infty} q_{x, y}=0 \text {, and } \sum_{z \in E: z \succeq y} \mu(y, z) Q\left(z,\{x\}^{\downarrow}\right) \geq 0, \quad x \neq y,
\end{array}\right.
$$

(iii) $Q^{*}$ is nonexplosive on $E$.

In this case, there exists a nonexplosive algebraic duality chain with the link kernel: $\Lambda(x, y)=I_{(y \preceq x)} \frac{\pi(y)}{H(x)}, x, y \in E$, and $Q^{*}$ in (3.1). Since the chain starts at $(0,0)$, (i) is satisfied and the dual chain starts at $(0,0)$. We will prove $Q$ in (4.1) also satisfies (ii). Obviously, $\lim _{x \rightarrow \infty} q_{x, y}=0$ for any $y \in E$. Let

$$
M(y, x)=\sum_{z \in E: z \succeq y} \mu(y, z) Q\left(z,\{x\}^{\downarrow}\right) .
$$

Using (2.2), we will calculate $M(y, x)$ for any $x, y \in E$, and show $M(y, x) \geq 0$ for any $x \neq y$.

(1) If $y=x+e_{i}$,

$$
\begin{aligned}
M\left(x+e_{i}, x\right) & =\sum_{z \in E: z \succeq x+e_{i}} \mu\left(x+e_{i}, z\right) Q\left(z,\{x\}^{\downarrow}\right) \\
& =\mu\left(x+e_{i}, x+e_{i}\right) Q\left(x+e_{i},\{x\}^{\downarrow}\right)=a_{i} .
\end{aligned}
$$


(2) If $y=x$,

$$
\begin{aligned}
M(x, x) & =\sum_{z \in E: z \succeq x} \mu(x, z) Q\left(z,\{x\}^{\downarrow}\right) \\
& =\mu(x, x) Q\left(x,\{x\}^{\downarrow}\right)+\sum_{i=1}^{2} \mu\left(x, x+e_{i}\right) Q\left(x+e_{i},\{x\}^{\downarrow}\right) \\
& =-\sum_{i=1}^{2} b_{i}+\sum_{i=1}^{2}(-1) a_{i}=-\sum_{i=1}^{2}\left(a_{i}+b_{i}\right) .
\end{aligned}
$$

(3) If $y=x-e_{i}$, for $j \neq i$,

$$
\begin{aligned}
M\left(x-e_{i}, x\right)= & \sum_{z \in E: z \succeq x-e_{i}} \mu\left(x-e_{i}, z\right) Q\left(z,\{x\}^{\downarrow}\right) \\
= & \mu\left(x-e_{i}, x-e_{i}\right) Q\left(x-e_{i},\{x\}^{\downarrow}\right) \\
& +\sum_{k=1}^{2} \mu\left(x-e_{i}, x-e_{i}+e_{k}\right) . \\
Q\left(x-e_{i}+e_{k},\right. & \left.\{x\}^{\downarrow}\right)+\mu\left(x-e_{i}, x+e_{j}\right) Q\left(x+e_{j},\{x\}^{\downarrow}\right) \\
= & 1 \cdot\left(-b_{j}\right)+\mu\left(x-e_{i}, x\right) Q\left(x,\{x\}^{\downarrow}\right) \\
& +\mu\left(x-e_{i}, x-e_{i}+e_{j}\right) \cdot Q\left(x-e_{i}+e_{j},\{x\}^{\downarrow}\right)+a_{j} \\
= & -b_{j}+(-1)\left(-b_{1}-b_{2}\right)+(-1) a_{j}+a_{j}=b_{i} .
\end{aligned}
$$

Similarly, we can calculate $M(y, x)=0$ for any other $x, y \in E$. Then we get $Q^{*}$ in (4.2), which is bounded obviously. Thus, $Q^{*}$ in (4.2) is nonexplosive and satisfies (iii). 


\section{Acknowledgement}

This work is supported by Beijing Natural Science Foundation (1194022), Premium Funding Project for Academic Human Resources Development in Beijing Union University (BPHR2020EZ01) and ZB10202001.

\section{References}

[1] D. Aldous and P. Diaconis, Shuffing cards and stopping times, American Mathematical Monthly 93(5) (1986), 333-348.

DOI: https://doi.org/10.1080/00029890.1986.11971821

[2] D. Aldous and P. Diaconis, Strong uniform times and finite random walks, Advances in Applied Mathematics 8(1) (1987), 69-97.

DOI: https://doi.org/10.1016/0196-8858(87)90006-6

[3] J. A. Fill, Time to stationarity for a continuous-time Markov chain, Probability in the Engineering and Informational Sciences 5(1) (1991), 61-76.

DOI: https://doi.org/10.1017/S0269964800001893

[4] P. Diaconis and J. A. Fill, Strong stationary times via a new form of duality, Annals of Probability 18(4) (1990), 1483-1522.

DOI: https://doi.org/10.1214/aop/1176990628

[5] P. Diaconis and J. A. Fill, Examples for the theory of strong stationary duality with countable state spaces, Probability in the Engineering and Informational Sciences 4(2) (1990), 157-180.

DOI: https://doi.org/10.1017/S0269964800001522

[6] J. A. Fill, Strong stationary duality for continuous-time Markov chains, Part I: Theory, Journal of Theoretical Probability 5(1) (1991), 45-69.

DOI: https://doi.org/10.1007/BF01046778

[7] Y. H. Mao and P. Zhao, Strong stationary duality for discrete time Möbius monotone Markov chains on $\mathbb{Z}_{+}^{d}$, Statistics and Probability Letters 123 (2017), 183-192.

DOI: https://doi.org/10.1016/j.spl.2016.12.010

[8] W. A. Massey, Stochastic orderings for Markov processes on partially ordered spaces, Mathematics of Operations Research 12(2) (1987), 350-367.

DOI: https://doi.org/10.1287/moor.12.2.350 
[9] P. Lorek and R. Szekli, Strong stationary duality for Möbius monotone Markov chains, Queueing Systems 71(1-2) (2012), 79-95.

DOI: https://doi.org/10.1007/s11134-012-9284-z

[10] P. Lorek and R. Szekli, Strong stationary duality for Möbius monotone Markov chains: Examples, Probability and Mathematical Statistics 36(1) (2016), 75-97.

[11] G. C. Rota, On the foundations of combinatorial theory I: Theory of Möbius functions, Zeitschrift für Wahrscheinlichkeitstheorie und Verwandte Gebiete 2(4) (1964), 340-368.

DOI: https://doi.org/10.1007/BF00531932

[12] P. Zhao, Siegmund duality for continuous time Markov chains on $\mathbb{Z}_{+}^{d}$, Acta Mathematica Sinica, English Series 34(9) (2018), 1460-1472.

DOI: https://doi.org/10.1007/s10114-018-7064-3 